\title{
Controlled Markov processes and \\ RELATED SEMIGROUPS OF OPERATORS
}

\author{
VOLF FRISHLING
}

A discrete time controlled Markov process is a family of measures, satisfying conditions similar to those of Markov processes. Each measure defines a strategy and each strategy defines a measure. If $T_{t}$ is a set of operators generated by the distribution of a state of the process at the moment $t$, then $T_{t}$ possesses a property analogous to the semi-group property, namely: $T_{t+s}=T_{t} \cdot T_{s}$. Here $(\cdot)$ is an operation between sets of operators. Such a family of sets is called a multiple semi-group. It is shown that under some natural restriction, a multiple semi-group generates a controlled Markov process.

This one-to-one correspondence makes it possible to study Markov processes through their semi-groups, in the same way as Markov processes have been studied. This novel approach leads to new proofs and interpretations of known results. For example, strauch's result on semiMarkovian strategies is obtained directly from the semi-group property.

The central problem in the theory of controlled processes is that of the existence of an optimal (E-optimal) strategy. Here it is shown that the optimal strategy is an extreme point of the set of all rewards. The set of all rewards satisfies an equation similar to Bellman's equation for the value function.

Received 5 October 1983. Thesis submitted to Flinders University of South Australia, September 1982 . Degree approved October 1983. Supervisor: Dr M. Jirina.

Copyright Clearance Centre, Inc. Serial-fee code: 0004-9727/83 $\$$ A2.00 +0.00 . 
The multiple semi-group technique allows to define continuous time controlled Markov processes. Results similar to those for the discrete time case are obtained for processes with bounded infinitesimal operators, that is for purely discontinuous processes.

The generalisation of these results to the case of arbitrary infinitesimal operators provides a method for the investigation of more general controlled Markov processes including diffusion.

The results obtained include those of Fleming for controlled diffusion processes. A theorem on the existence of a weak solution to a stochastic differential equation is proved as a side result.

\author{
I116 Argyle Street, \\ East Bentleigh, \\ Victoria 3165 , \\ Australia.
}

